Article

\title{
Ideal Coulomb Plasma Approximation in Line Shape Models: Problematic Issues
}

\author{
Joel Rosato *, Hubert Capes and Roland Stamm
}

Aix Marseille Université, CNRS, PIIM UMR 7345, Marseille 13397, France;

E-Mails: h.capes@laposte.net (C.H.); roland.stamm@univ-amu.fr (S.R.)

* Author to whom correspondence should be addressed; E-Mail: joel.rosato@ univ-amu.fr; Tel.: +33-49128-8624.

Received: 23 March 2014; in revised form: 11 June 2014 / Accepted: 13 June 2014 /

Published: 19 June 2014

\begin{abstract}
In weakly coupled plasmas, it is common to describe the microfield using a Debye model. We examine here an "artificial" ideal one-component plasma with an infinite Debye length, which has been used for the test of line shape codes. We show that the infinite Debye length assumption can lead to a misinterpretation of numerical simulations results, in particular regarding the convergence of calculations. Our discussion is done within an analytical collision operator model developed for hydrogen line shapes in near-impact regimes. When properly employed, this model can serve as a reference for testing the convergence of simulations.
\end{abstract}

Keywords: Stark broadening; hydrogen plasmas; numerical simulations; collision operators

\section{Introduction}

The Spectral Line Shapes in Plamas Code Comparison Workshop (SLSP) [1] focuses on a set of standardized physical problems to be addressed using codes from different research groups/labs. Amongst these problems is the description of Stark line shapes with ion dynamics effects, referred to as the cases " 1 " and " 2 " in the first (2012) and second (2013) editions of the Workshop. In order to get a simple interpretation of what the codes effectively calculate, a set of idealizing assumptions has been considered. For example, the electrons and the ions are assumed to move along straight path trajectories and they produce unscreened Coulomb potentials (ideal plasma approximation). The purpose of this paper is to show that the latter assumption raises a problem of consistency in the interpretation 
of "ab initio" numerical simulations, i.e., simulations that are free from physical approximations in the evaluation of the plasma microfield and in the calculation of the atomic dipole autocorrelation function. They commonly serve as a reference for testing other models. Although very convenient in practice (in particular for programming purposes), the simulations can take a long time and become useless in the case where a large amount of particles is required in the evaluation of the microfield. This occurs for weakly coupled plasmas, when the Debye length is much larger than the mean interparticle distance. A relevant strategy when performing a simulation is to take a box (either of a cubic, spherical, or more complex shape) of characteristic size of the order of the Debye length (typically larger by a factor of several units), which means that the number of particles can be very large in weakly coupled plasma conditions. If the plasma is so weakly coupled that a calculation cannot be performed on a reasonable time scale, the box size is reduced and the corresponding number of particles is adjusted in such a way that a relevant statistical quantity (like the microfield probability density function or the microfield autocorrelation function) is well reproduced within a few percents error bars. We suggest that this procedure does not suffice to obtain reference profiles in the case of infinite Debye length. The neglect of far perturbers may result in a significant underestimate of the line broadening if the microfield is dynamic. Our discussion is based on the use of an analytical model for Stark broadening in regimes such that the impact approximation is not far from being satisfied by the perturbers under consideration (ions or electrons) [2,3]. We consider the Lyman $\alpha$ line broadened due to the electrons only at $N=10^{17} \mathrm{~cm}^{-3}$ and $T=100 \mathrm{eV}$ (subcase "1.1.3.1.1" of the 2013 SLSP), and we assume an unscreened Coulomb electric field as required in the Workshop statement of cases.

\section{A Collision Operator Model for the $\lambda_{D} \rightarrow \infty$ Limit}

The plasma conditions yield a value of about $4 \times 10^{-3}$ for the ratio $b_{W} / r_{0}$ between the Weisskopf radius $\left(b_{W}=\hbar n^{2} / m_{e} v\right.$, where $n=2$ is the upper principal quantum number and $v=\sqrt{2 T / m_{e}}$ is the electrons' thermal velocity) and the mean interparticle distance $\left(r_{0}=N^{-1 / 3}\right)$, which suggests that a collision operator may be used. The standard impact models for hydrogen (e.g., Griem et al. [4,5]) and their extensions (e.g., the Lewis cut-off [6] and the unified theory [7,8]) account for particle correlations within a Debye screening model and, hence, are not compatible with the unscreened electric field assumption $\left(\lambda_{D} \rightarrow \infty\right)$ done in the subcase 1.1.3.1.1. This "artificial" setting of an infinite Debye length requires a careful reconsideration of the role of far perturbers (weak collisions), in a more general framework than that involved in the standard collision operator models. Recently, it has been shown that the perturbers that effectively contribute to the line broadening are those which are located at a distance smaller than $v / \bar{\gamma}$, where $\bar{\gamma}$ is the line's characteristic width, because of the finite lifetime of the emitter (see the discussions in [2,3]). This length may be interpreted as an upper cut-off in place of the Debye length for our "artificial" Coulomb plasma. Applying the model reported in [2] to the subcase 1.1.3.1.1 yields the following formula for the line shape

$$
I(\Delta \omega)=\frac{1}{\pi} \operatorname{Re} \frac{1}{-i \Delta \omega+\gamma(\Delta \omega)}
$$


where $\Delta \omega$ is the frequency detuning and where $\gamma(\Delta \omega)$ is the collision operator taken in the bracket $\langle 2,1,0|\ldots| 2,1,0\rangle$ (spherical base). The latter is given by $\left\{\right.$ see [2], Equation (A4), setting $\left.\lambda_{D} \rightarrow \infty\right\}$

$$
\gamma(\Delta \omega)=\frac{9 g}{8 \sqrt{\pi}} \int_{0}^{\infty} d u e^{-u}\left[\sqrt{\frac{\pi u}{-i \widetilde{\Delta \omega}}} \operatorname{erf}\left(\sqrt{\frac{-i \widetilde{\Delta \omega}}{u}}\right)+E_{1}\left(\frac{-i \widetilde{\Delta \omega}}{u}\right)\right]
$$

We have used here a system of units such that the Weisskopf radius $b_{W}$ and the thermal velocity $v$ are equal to unity; $g=4 \pi N b_{W}^{3} / 3$ is the number of particles in the Weisskopf sphere, which is much smaller than unity (namely, of the order of $2 \times 10^{-7}$ here), and the quantity $\widetilde{\Delta \omega}$ is defined as $\Delta \omega+i \theta \gamma_{0}$ where $\gamma_{0} \equiv \gamma(\Delta \omega=0)$ and $\theta$ is an atomic physics factor of the order of unity. In our calculations we have set $\theta=1$. Like standard collision operators, $\gamma(\Delta \omega)$ exhibits a decomposition into a strong collision part (the first term of the right-hand side) and a weak collision part (the second term). It is worth noting that Equation (2) does not provide a closed expression for $\gamma(\Delta \omega)$, because of the presence of $\gamma_{0}$ (through the $\widetilde{\Delta \omega}$ term) in the error and exponential integral functions; instead, it implies an equation of the form $\gamma_{0}=F\left(\gamma_{0}\right)$ where $F$ is a nonlinear function. In [2], this equation was solved numerically by iterations and it was shown that this method is quickly convergent.

\section{Questioning the Validity of Simulations}

Our Equation (1) applied to the subcase 1.1.3.1.1 leads to a line shape about twice larger than that obtained from the simulation results that have been presented during the Workshop. These simulations assume a finite box that contains several thousands of particles. According to the model, we suggest that this deviation stems from the neglect of the far perturbers (i.e., those inside the $v / \bar{\gamma}$ sphere but outside the simulation box) in the simulations. It is quite difficult to test this argument by enlarging the simulation box up to $v / \bar{\gamma}$ because this would imply a very large number of particles, up to several billions. Instead, we have modified our model [2] in such a way to account for a finite plasma size $R$. The general collision operator formula accounting for finite Debye length $\{[2]$, Equation (A4) $\}$ has been used with the formal substitution $\lambda_{D} \equiv R$.

Figure 1 shows an application to a cubic box of size $R=3400 b_{W}$. The three plots correspond to (i) the result of the model assuming finite $R$ (referred to as "UTPP with box cut-off"); (ii) the model assuming infinite $R$ ("UTPP"); and (iii) a numerical simulation performed with our code [9]. The simulation has been performed using about 2000 particles, which corresponds to the setting of $R=3400 b_{W}$ in the plasma conditions associated with the subcase 1.1.3.1.1. The dipole autocorrelation function has been evaluated assuming 5000 histories, with a time step of about $0.08 r_{0} / v$. The setting of a smaller time grid yields no significant modification in the results. In order to get a dipole autocorrelation function sufficiently close to zero at large times, we have taken $10^{6}$ time steps. Our simulation (not presented at the Workshop 2013 edition) is in agreement with the other simulation codes presented at the Workshop [10]. These codes also assumed several thousands of particles. As can be seen in the figure, the model accounting for the box size yields a line shape that coincides with the simulation result (up to the statistical noise), whereas the model that assumes infinite $R$ yields a line shape about twice larger. The setting of several thousands of particles in the simulations presented at the Workshop was supported by the reproducibility of plasma statistical quantities (such as the microfield probability density function). 
However, this does not mean that all of the line broadening is taken into account. The line width has a logarithmic dependence on $R$ up to a threshold of the order of $v / \bar{\gamma}$ (see Figure 2), which is much larger than the box size considered in the simulations. This suggests that the simulations performed at the workshop have not converged in the plasma conditions (subcase "1.1.3.1.1") considered in our investigation. Note, we cannot provide such a strong statement in other cases where our collision operator model is not applicable; a (straightforward) convergence test should involve a plot of the simulated line width in terms of $R$.

Figure 1. Hydrogen Lyman $\alpha$ line shape calculated according to the subcase 1.1.3.1.1. The three plots correspond to (i) the result of the model assuming a finite box size $R$ (dashed line); (ii) the model assuming infinite $R$ (solid line); and (iii) a numerical simulation (circles). The second plot (solid line) serves as a reference. Keeping finite $R$ results in an underestimate of the line width.

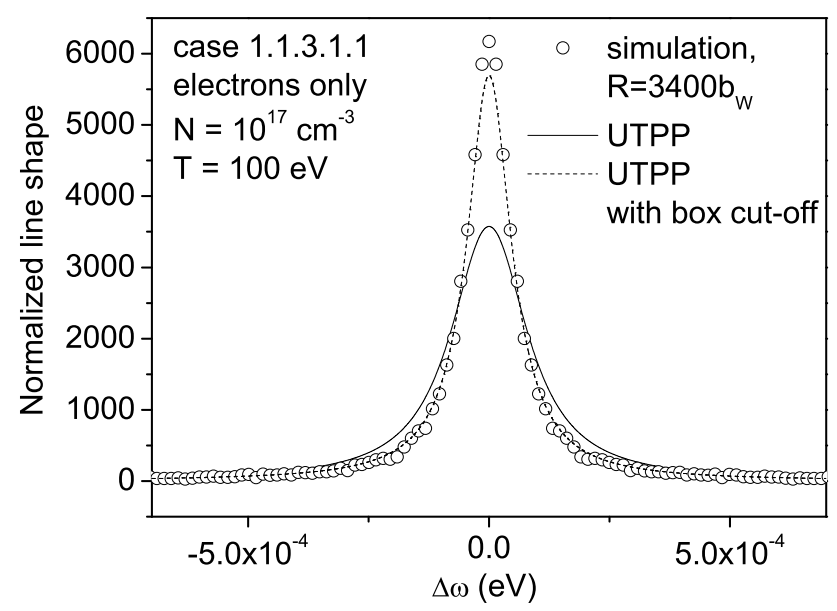

Figure 2. The collision operator at the line center $(\Delta \omega=0)$ is a weakly increasing function of the box size $R$ and saturates when $R$ is of the order of $10^{8}$. This roughly corresponds to the value of $v / \bar{\gamma}$ (see text).

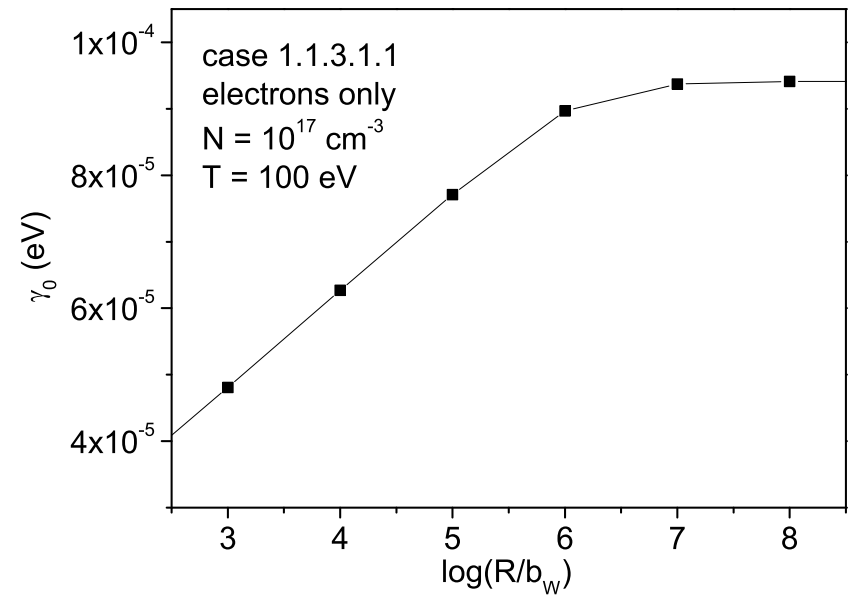




\section{Conclusions}

In conclusion, we have shown that an artificial setting of an infinite Debye length in numerical simulations requires a careful interpretation of the results. With an analytical model, we have shown that the simulations may neglect a considerable amount of perturbers that effectively contribute to the broadening; the deviations between the simulations and the analytical model are sufficiently large (up to a factor of 2) so that they must deserve a special analysis. The ideal plasma conditions considered in this work were motivated by the need of a physical model sufficiently simple so that line shape codes results can be easily compared (SLSP Workshop). Our results suggest that this physical model is not appropriate; a more relevant one should retain particle correlations within a Debye field. The analytical line shape model used in our work is restricted to weakly coupled plasmas in conditions such that the microfield evolves at a time scale much shorter than the time of interest. A further investigation should focus on an extension able to account for more general cases, e.g., when the emitter suffers simultaneous strong collisions (namely, when the ratio $b_{W} / r_{0}$ is significant).

\section{Acknowledgments}

This work was carried out within the framework of the European Fusion Development Agreement and the French Research Federation for Fusion Studies. It is supported by the European Communities under the contract of Association between Euratom and CEA. The views and opinions expressed herein do not necessarily reflect those of the European Commission.

\section{Conflicts of Interest}

The authors declare no conflict of interest.

\section{References}

1. Stambulchik, E. Review of the 1st Spectral Line Shapes in Plasmas code comparison workshop. High Energy Density Phys. 2013, 9, 528-534.

2. Rosato, J.; Capes, H.; Stamm, R. Influence of correlated collisions on Stark-broadened lines in plasmas. Phys. Rev. E 2012, 86, 046407:1-046407:8.

3. Rosato, J.; Capes, H.; Stamm, R. Divergence of the Stark collision operator at large impact parameters in plasma spectroscopy models. Phys. Rev. E 2013, 88, 035101:1-035101:3.

4. Griem, H.R.; Kolb, A.C.; Shen, K.Y. Stark broadening of hydrogen lines in a plasma. Phys. Rev. 1959, 116, 4-16.

5. Griem, H.R.; Blaha, M.; Kepple, P.C. Stark-profiles calculations for Lyman-series lines of one-electron ions in dense plasmas. Phys. Rev. A 1979, 19, 2421-2432.

6. Lewis, M. Stark broadening of spectral lines by high-velocity charged particles. Phys. Rev. 1961, $121,501-505$.

7. Voslamber, D. Unified model for Stark broadening. Z. Naturforsch. 1969, 24a, 1458-1472.

8. Smith, E.W.; Cooper, J.; Vidal, C.R. Unified classical-path treatment of Stark broadening in plasmas. Phys. Rev. 1969, 185, 140-151. 
9. Rosato, J.; Marandet, Y.; Capes, H.; Ferri, S.; Mossé, C.; Godbert-Mouret, L; Koubiti, M; Stamm, R. Stark broadening of hydrogen lines in low-density magnetized plasmas. Phys. Rev. E 2009, 79, 046408:1-046408:7.

10. Ferri S.; Calisti A.; Mossé C.; Rosato J.; Talin B.; Alexiou S.; Gigosos M.A.; González M. A.; González D. Ion dynamics effect on Stark broadened line shapes: A cross comparison of various models Atoms, submitted.

(C) 2014 by the authors; licensee MDPI, Basel, Switzerland. This article is an open access article distributed under the terms and conditions of the Creative Commons Attribution license (http://creativecommons.org/licenses/by/3.0/). 